INRA Prod. Anim., 2000, 13 (4), 269-276
M. SANCRISTOBAL-GAUDY ${ }^{1}$, G. RENAND ${ }^{2}$, Y. AMIGUES ${ }^{3}$, M.-Y. BOSCHER ${ }^{3}$,

H. LEVÉZIEL ${ }^{4}, B$. BIBÉ ${ }^{5}$

1 I NRA Laboratoi re de Généti que Cellulai re, BP 27, 31326 Castanet Tolosan Cedex

2 I NRA Stati on de Généti que Quanti tati ve et Appli quée, 78352 J ouy-en-j osas Cedex 3 LABOGENA, 78352 J ouy-en-J osas Cedex 4 INRA Laboratoi re de Généti que Bi ochi mi que et de Cytogénéti que, 78352 J ouyen-J osas Cedex

5 INRA Département de Généti que Ani male BP 27, 31326 Castanet Tolosan Cedex

Courrie : msc@toulouseinra.fr

\section{Traçabilité individuelle des viandes bovines à l'aide de marqueurs génétiques}

\begin{abstract}
Des tests d'ADN, tels que ceux employés en médecine légale, peuvent être utilisés à des fins de traçabilité individuelle des animaux et des produits dérivés, la viande notamment. Cette approche, dans un contexte économique difficile lié à la crise de la «vache folle», va permettre de sécuriser et de renforcer la nouvelle réglementation sur l'étiquetage des viandes en suivant l'identification des bovins de l'étable à l'étal.
\end{abstract}

\begin{abstract}
Depuis le début des années 80 on constate, en France comme dans le reste de l'E urope, une réduction significative de la consommation de viande bovine dont les causes sont multiples (Potherat et Mainsant 1995, Combris 1997). Les consommateurs exigent maintenant une meilleure information sur la qualité nutritionnelle et sanitaire des produits qui leur sont proposés. Cette exigence implique que l'origine de la viande soit garantie, c'est-àdire qu'il y ait tracabilité continue depuis l'animal jusqu'aux pièces de viande commer-
\end{abstract}

\section{Résumé}

E $\mathbf{n}$ réponse à la crise de confiance des consommateurs et leur régulière désaffection pour la viande bovine, et dans le cadre des efforts développés actuellement pour améliorer et sécuriser la traçabilité des bovins, nous avions pour objectif de proposer et de valider une méthode d'identification individuelle des viandes bovines à l'aide de marqueurs moléculaires de type microsatellite. Plusieurs prélèvements ont été effectués sur des jeunes bovins de domaines expérimentaux INRA, in vivo et post mortem. La détermination des génotypes aux 11 loci, puis la comparaison deux à deux de ces génotypes à l'aide d'une méthode statistique adéquate prenant en compte les particularités des microsatellites permet de recommander d'une part que le typage soit réalisé sur au moins huit marqueurs pour assurer une traçabilité parfaite, et d'autre part que des précautions indispensables soient prises concernant la qualité des prélèvements et la gestion des échantillons lors d'une mise en œuvre à grande échelle. cialisées. C'est ainsi qu'ont été mis en place des accords interprofessionnels et des réglementations administratives pour l'ensemble de la filière, d'abord à l'échelle nationale puis de la communauté européenne, de même que des cahiers des charges spécifiques à certaines filières d'approvisionnement du type Label Rouge ou enseignes de grande distribution, pour que l'information portée sur les étiquettes soit claire et compréhensible par les consommateurs et permette toute vérification de l'exactitude de cette information.

Depuis de nombreuses années un système d'identification des bovins et des cheptels fonctionne en France dans un but de suivi zootechnique et sanitaire des animaux et des élevages. Suite au règlement $C E n^{\circ} 820 / 97$, cette exigence fut étendue à tous les pays de la communauté et a conduit à la mise en place d'une nouvelle identification pérenne généralisée (IPG) de tous les bovins nés ou élevés en France avec constitution d'une base de données centralisées. Ces outils (double bouclage, registre bovin, passeport, etc.) permettent d'identifier et de suivre tout animal, ainsi que son statut sanitaire, présent en France de sa naissance (ou son entrée en France) à son abattage (ou sa sortie de France) quels que soient le ou les détenteurs successifs. 
Si l'existence d'un système d'identification des bovins en France a permis une réaction rapide à la crise de l'ESB en 1996 en indiquant l'origine française des viandes (VBF), l'interprofession a décidé en février 1997 d'aller plus loin en signant un accord pour qu'au minimum figurent les trois informations suivantes sur l'étiquette des viandes bovines : origine (animal né, élevé et abattu en F rance), catégorie (J eune Bovin, Taureau, Génisse ou Vache) et type racial (Laitier, à Viande ou Mixte).

Si cet étiquetage a pour objectif d'informer le consommateur sur l'origine de la viande qui lui est proposée, il est indispensable de mettre en place un système qui lui garantisse la fiabilité des informations indiquées. La traçabilité ainsi définie doit permettre aux services de contrôle de retrouver et d'apporter la preuve, a posteriori, de la véracité des informations en remontant jusqu'au numéro d'identification de l'animal et à l'élevage de provenance.

De la naissance de l'animal à la pièce de viande, le suivi de l'identification se trouve confronté à un certain nombre de contraintes. Tant que l'animal est encore vivant, I'IPG assure une traçabilité théoriquement sans faille du fait d'un numéro d'identification unique, de la pose de deux boucles, de l'édition d'un passeport et de l'obligation faite à chaque nouveau détenteur (éleveur, transporteur, abatteur) de vérifier la concordance entre les numéros d'identification portés sur les boucles et sur le passeport. A l'abattoir, il est par contre techniquement impossible de maintenir cette IPG sur les carcasses et les pièces de viande préparées en atelier de découpe. Les carcasses sont en effet repérées à partir d'un numéro de tuerie et d'une date d'abattage, alors qu'un numéro de lot est attribué aux pièces de viande. Les entreprises d'abattage et de découpe ont dû réorganiser en profondeur leurs méthodes de travail afin d'éviter le mélange des pièces de viande issues d'animaux différents (Dupit 1998) et d'assurer la correspondance entre les différents identifiants utilisés.

Tout au long de la filière peuvent donc surgir des difficultés liées à des opérations de saisie d'information ou de transfert et de vérification d'information, qui constituent donc des maillons faibles si on considère l'ensemble des précautions nécessaires à une parfaite traçabilité comme une chaîne à ne pas interrompre (Chaisemartin 1998).

La traçabilité ne peut être garantie que s'il existe un système de vérification et de contrôle tout au long de la chaîne. Différentes techniques existent permettant de vérifier a posteri ori les informations portées sur les étiquettes. L'origine géographique, ainsi que le type d'alimentation, devraient pouvoir être déterminés à partir de méthodes analytiques. C'est ainsi que la détermination des teneurs en isotopes stables, méthode actuellement appliquée aux végétaux, devrait permettre d'authentifier le lieu de production d'animaux réputés nourris avec des ressources locales, eau de boisson et végétaux (Renou 1998). L'analyse des composés volatils est également un puissant moyen de caractérisation car elle permet d'obtenir une «signature» des produits (Thonat et al 1999). En effet, après constitution d'une banque de signatures caractéristiques de différents types de production, il est possible de comparer la signature d'un produit donné avec cette banque et de reconnaître l'origine en appliquant une modélisation statistique dite des réseaux de neurones. Ces méthodes analytiques font l'objet de recherches poussées à la Station de Recherches sur la Viande de I'INRA de Theix afin de mettre au point des techniques de mesure rapides qui soient utilisables dans de nombreux secteurs de l'agro-alimentaire. Toutefois, cette approche tient plus d'une vérification statistique que d'une traçabilité individuelle.

S'il est facile de vérifier le sexe de l'animal à partir d'un échantillon de viande par la mise en évidence de marqueurs ADN du chromosome Y (Léonard et al 1987), il n'est pas envisageable de préciser le type d'animal. D'autre part, l'analyse des fréquences des différents allèles de marqueurs ADN du type microsatellite a permis de caractériser de nombreuses races bovines et de montrer qu'il est possible de les discriminer (Moazami-Goudarzi et al 1997). Connaissant les profils caractéristiques de ces races, il est possible d'assigner un individu à l'une d'entre elles en comparant son propre profil aux profils moyens de ces races. Cette approche repose également sur une vérification statistique par des calculs de probabilité d'appartenance, distances génétiques, réseaux de neurones, etc. (Cornuet et al 1996, Ollivier et al 2000). Ces marqueurs sont neutres vis-à-vis de l'évolution des races puisqu'ils ne correspondent pas à des gènes codants. Ils sont utilisés en attendant l'identification de gènes marqueurs responsables d'un phénotype caractéristique de la race. C'est ainsi que des recherches sont actuellement menées sur les gènes de la coloration de la robe des bovins dans un but de détermination raciale (Rouzaud et al 2000).

Toutes ces méthodes de vérification a posteri ori de l'origine ou de la race de l'animal dont est issue la pièce de viande expertisée sont basées sur la comparaison d'un profil pour une série de caractéristiques plus ou moins discriminantes avec des profils de référence déterminés au préalable. De ce fait les réponses sont du type probabiliste et, si elles permettent de vérifier la vraisemblance de l'information générique portée sur les étiquettes, elles ne permettent pas de vérifier l'intégrité de l'identification tout au long de la filière. II convenait donc de mettre en place une méthode qui pallie ces inconvénients.

Tant que le matériel moléculaire (ADN) n'est pas dégradé, la séquence génétique d'un individu, unique et inaltérable, peut être utilisée comme l'identifiant individuel qui assure 
une traçabilité parfaite, indépendante des aléas de saisie ou de transfert d'information. II est bien évidemment illusoire de vouloir connaître la séquence génétique complète de chaque animal, mais il est possible d'envisager l'identification des animaux à partir d'un nombre restreint de régions du génome dont le typage soit facile et automatisable. Ces régions (loci) doivent être polymorphes et en nombre suffisant pour assurer que chaque individu soit caractérisé d'une manière unique parmi l'ensemble de la population de l'espèce. Cette approche, connue sous le nom de test d'ADN, est de plus en plus utilisée en médecine légale (voir par exemple Balding et Nichols 1995).

Cet article présente les résultats d'une expérience réalisée dans le cadre d'un contrat entre Interbev et le CIV (Centre d'Information sur les Viandes) d'une part, I'INRA et LABOGENA d'autre part, pour mettre au point et valider une méthode d'identification individuelle des bovins (animaux et viande) à l'aide de marqueurs génétiques moléculaires.

\section{1 / Matériel et méthodes}

De l'ADN a été extrait de différents prélèvements sur animaux et sur viande pour typer les allèles d'un certain nombre de marqueurs. Un marqueur est un segment de la molécule d'ADN qui peut présenter diverses formes ou allèles (polymorphisme) dans la population. Le nombre et la fréquence des allèles est variable selon le marqueur considéré et la population concernée. Les marqueurs de type microsatellite présentent l'intérêt d'avoir souvent un fort polymorphisme. Pour chaque marqueur, un individu possède deux allèles, un transmis par son père et un transmis par sa mère, qui déterminent son génotype à ce marqueur. Sur un ensemble de marqueurs, la combinaison des paires d'allèles typés permet de déterminer un génotype propre à chaque individu pour ces marqueurs et donc de le caractériser.

En prenant en compte la fiabilité des techniques de génétique moléculaire, il convient de trouver le nombre de marqueurs à utiliser pour que les génotypes mis en évidence soient suffisamment représentatifs de l'ensemble du génome des animaux étudiés afin d'éviter toute erreur d'identification et d'assurer ainsi une traçabilité parfaite.

\section{1 / Les prélèvements}

Différents prélèvements ont été effectués sur 144 jeunes bovins des domaines INRA de Bourges et du Pin-au-Haras, en majorité de race charolaise, dont certains étaient demifrères. Six types de prélèvements ont été réalisés dans cette étude : sur l'animal en vif (sang, poils) et après abattage avec deux degrés de maturation de la viande (24 heures, 15 jours) et deux méthodes de prélèvement (au scalpel et à l'aiguille à biopsie à titre de test). Le nombre total des prélèvements s'élève à 552 .

\section{2 / Les typages}

Compte tenu de la diversité du matériel biologique utilisé, il a été nécessaire d'adapter les méthodes d'extraction de l'ADN à chaque type de prélèvement (tableau 1).

Tableau 1. Méthodes d'extraction de l'ADN adaptées à chaque type de prélèvement.

\section{Sang}

Lavage ( 2 heures à $4^{\circ} \mathrm{C}$ ) de $1 \mathrm{ml}$ de sang avec $4 \mathrm{ml}$ d'une solution de $\mathrm{NE}(\mathrm{NaCl} 10 \mathrm{mM}$, EDTA $10 \mathrm{mM}$ ) et récupération du culot des leucocytes par centrifugation. L'opération est renouvelée deux fois. Lyse alcaline des leucocytes par une solution de $\mathrm{NaOH} 200 \mathrm{mM}\left(2\right.$ heures à $65^{\circ} \mathrm{C}$ ).

Neutralisation par une solution de $\mathrm{HCl} 200 \mathrm{mM}$, Tris- $\mathrm{HCl} 100 \mathrm{mM}$ :

\section{Poils}

A partir des bulbes de trois poils ajouter directement le $\mathrm{NaOH}$ ( 1 heure à $65^{\circ} \mathrm{C}$ ).

Neutraliser avec la solution $\mathrm{HCl}$, Tris- $\mathrm{HCl}$.

\section{Viande}

A partir de 5 à $10 \mathrm{mg}$ de viande ajouter directement le $\mathrm{NaOH}\left(2\right.$ heures à $65^{\circ} \mathrm{C}$ ).

Neutraliser avec la solution $\mathrm{HCl}, \mathrm{Tris}-\mathrm{HCl}$.

Onze marqueurs de type microsatellite ont été sélectionnés parmi ceux qui ont déjà été utilisés dans divers programmes de recherche de QTL, de diversité génétique ou de contrôle de paternité. Génétiquement indépendants, polymorphes, aptes à être multiplexés (analysés conjointement) et analysés sur un séquenceur automatique, leur qualité de typage devait être assurée. Ces onze marqueurs microsatellites ont été amplifiés lors d'une seule réaction de $P C R$ en utilisant des amorces marquées avec trois fluorochromes différents permettant de détecter simultanément tous les produits d'amplification (voir encadré). Les produits de PCR sont analysés par un séquenceur automatique $A B I 310$ et les profils obtenus pour les differents marqueurs sont interprétés à l'aide des logiciels GENESCAN et GENOTYPER pour déterminer les génotypes qui sont transférés dans une base de données permettant la comparaison entre les échantillons.

Les typages des 552 échantillons provenant des 144 bovins ont été réalisés en aveugle à LABOGENA, de manière à prendre en compte les éventuelles erreurs de typage inhérentes à ce type de marqueur.

\section{3 / L'approche statistique}

Une approche classique dite du maximum de vraisemblance a été adoptée dans cette étude. L'analyse statistique des résultats a été réalisée sur les génotypes obtenus lors d'un seul typage pour prendre en compte d'éventuelles erreurs de typage. 


\section{E mpreintes génétiques obtenues à l'aide des profils électrophorétiques de marqueurs microsatellites.}

Trois échantillons (sang, poil et viande) appartenant à un même animal ont été analysés à l'aide de 11 marqueurs microsatellites. Chaque marqueur se situe dans une gamme de taille bien précise et est marqué à l'aide d'une molécule fluorescente bleue (6-FAM), verte (TET) ou jaune (HEX). Marqueur Fluorochrome

$\begin{array}{lc}\text { TGLA 227 } & \text { TET } \\ \text { INRA 222 } & \text { HEX } \\ \text { INRA 072 } & \text { 6-FAM } \\ \text { ILSTS 065 } & \text { TET } \\ \text { INRA 092 } & 6 \text {-FAM } \\ \text { ETH 225 } & \text { HEX } \\ \text { INRA 135 } & \text { TET } \\ \text { INRA 177 } & \text { HEX } \\ \text { BM 1824 } & \text { 6-FAM } \\ \text { HUJI 177 } & \text { 6-FAM } \\ \text { SPS 115 } & \text { HEX }\end{array}$

La courbe rouge correspond à un standard de

Profils obtenus dans trois prélèvements avec les 4 microsatellites marqués avec le fluorochrome 6-FAM :
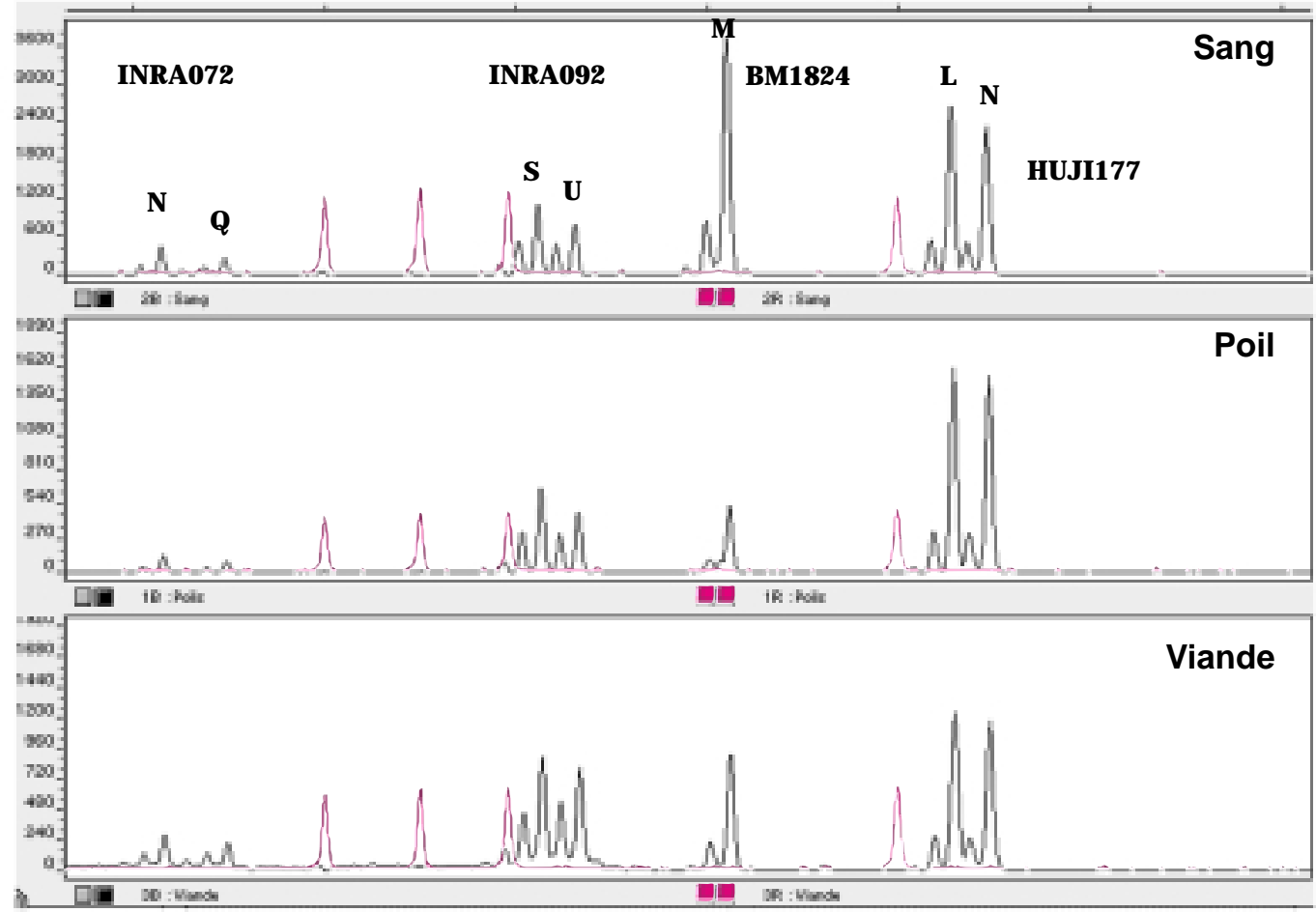

taille qui permet de déterminer la taille exacte de chacun des allèles des différents marqueurs. Les tailles des allèles ainsi obtenus sont transcrites en terme d'allèle lettre (exemple sur le profil 'Sang') et sont, après importation dans une base de données informatique, facilement exploitables pour les traitements automatiques de compa-raison d'échantillons.

Marqueur Allèle au marqueur

$\begin{array}{lccc} & \text { Sang } & \text { Poil } & \text { Viande } \\ \text { TGLA 227 } & \text { KO } & \text { KO } & \text { KO } \\ \text { INRA 222 } & \text { HL } & \text { HL } & \text { HL } \\ \text { INRA 072 } & \text { NQ } & \text { NQ } & \text { NQ } \\ \text { ILSTS 065 } & \text { LP } & \text { LP } & \text { LP } \\ \text { INRA 092 } & \text { SU } & \text { SU } & \text { SU } \\ \text { ETH 225 } & \text { OO } & \text { OO } & \text { OO } \\ \text { INRA 135 } & \text { JM } & \text { JM } & \text { JM } \\ \text { INRA 177 } & \text { PP } & \text { PP } & \text { PP } \\ \text { BM 1824 } & \text { MM } & \text { MM } & \text { MM } \\ \text { HUJ I 177 } & \text { LN } & \text { LN } & \text { LN } \\ \text { SPS 115 } & \text { LL } & \text { LL } & \text { LL }\end{array}$

Etant donné les génotypes à 11 loci de 2 échantillons, nous voulons tester I'hypothèse $\mathrm{H} 0$ : «les 2 échantillons proviennent du même individu » contre l'hypothèse $\mathrm{H} 1$ : «les 2 échantillons proviennent de 2 individus quel- conques ». En notant L0 (respectivement L1) la vraisemblance des données sous I'hypothèse $\mathrm{HO}$ (respectivement $\mathrm{H} 1$ ), la valeur du rapport $\mathrm{L} 1 / \mathrm{L} 0$ va permettre de déclarer vraie soit HO, soit H1. 
La qualité du test sera mesurée selon deux critères : la probabilité de décider que deux échantillons du même animal proviennent de deux individus différents d'une part (c'est-àdire accepter I'hypothèse $\mathrm{HI}$ alors que c'est $\mathrm{HO}$ qui est vraie), et la probabilité de décider que deux échantillons provenant de deux individus différents sont issus du même individu (c'est-à-dire accepter $\mathrm{HO}$ alors que $\mathrm{H} 1$ est vraie) d'autre part ; ces deux quantités doivent être quasiment nulles. Ces tests statistiques ont porté sur 548 prélèvements issus des 140 animaux ayant au moins deux prélèvements.

\section{2 / Résultats}

\section{1 / Qualité de l'ADN}

De l'ADN de bonne qualité a été extrait de toutes les catégories d'échantillons du protocole, notamment de la viande après 15 jours de maturation, prélevée ou non à l'aiguille à biopsie, et du sang. Seuls les échantillons de poils ont nécessité plus de travail. Le nombre d'extractions ayant dû être refaites pour obtenir de l'ADN analysable est présenté dans le tableau 2 selon le type de prélèvement.

Tableau 2. Bilan des extractions d'ADN selon le type de prélèvement.

\begin{tabular}{|l|c|c|}
\hline $\begin{array}{l}\text { Type de } \\
\text { prélèvement }\end{array}$ & $\begin{array}{c}\text { Nombre } \\
\text { total }\end{array}$ & $\begin{array}{c}\text { Extractions } \\
\text { refaites }\end{array}$ \\
\hline Sang & 110 & $7(6,4 \%)$ \\
Poils & 140 & $31(22,1 \%)$ \\
Viande & 302 & $2(0,7 \%)$ \\
\hline
\end{tabular}

Sur les onze marqueurs utilisés, un seul a présenté des problèmes lors de son amplification par PCR. Pour les dix autres marqueurs les défauts d'amplification sont faibles $(1,1 \%$ en moyenne) et dépendent en fait du type de prélèvement (tableau 3), avec une fréquence plus élevée pour les prélèvements de viande à l'aiguille à biopsie et pour les poils. Les échantillons sanguins et de viande prélevée au scalpel présentent très peu de problème technique d'amplification.

Tableau 3. Défauts d'amplification selon le type de prélèvement (tous les marqueurs, sauf le 11).

\begin{tabular}{|l|c|cc|}
\hline $\begin{array}{l}\text { Type de } \\
\text { prélèvement }\end{array}$ & $\begin{array}{c}\text { Nombre } \\
\text { d'échantillons }\end{array}$ & \multicolumn{2}{|c|}{$\begin{array}{c}\text { Défauts } \\
\text { d'amplification }\end{array}$} \\
\hline Sang & 110 & 2 & $(0,2 \%)$ \\
Poils & 140 & 25 & $(1,8 \%)$ \\
Viande 24h scalpel & 126 & 1 & $(0,1 \%)$ \\
Viande 24h aiguille & 48 & 17 & $(3,5 \%)$ \\
Viande 15j scalpel & 84 & 2 & $(0,2 \%)$ \\
Viande 15j aiguille & 44 & 15 & $(3,4 \%)$ \\
\hline
\end{tabular}

(1) Pourcentage calculé en rapportant le nombre de défauts au nombre total d'amplifications (= nombre d'échantillons $\times 10$ marqueurs).
La qualité de I'ADN extrait des différents échantillons est donc très satisfaisante. Les meilleurs résultats sont toutefois obtenus pour les échantillons de sang et de viande prélevée au scalpel qui nécessitent en définitive moins de travail pour obtenir de l'ADN amplifié de bonne qualité.

\section{2 / Fiabilité des typages}

Les différents échantillons d'un même animal ont été comparés deux à deux à l'aide du test statistique, sur l'ensemble des animaux ayant au moins deux prélèvements (548 prélèvements utilisables issus de 140 individus). Des erreurs de typage ont ainsi pu être détectées, elles sont présentées dans le tableau 4 pour chacun des marqueurs. Le taux global de génotypes faussement déterminés est égal à $0,8 \%$ Le taux global d'allèles faussement déterminés est égal à $0,4 \%$. L'ensemble des résultats de typage est donc très satisfaisant. II est à noter qu'en fait la majorité (73\%) des erreurs de typage est liée à la présence d'allèles quasi-nuls (un allèle n'est pas détecté car masqué par l'autre lors de la lecture du gel), ce qui est parfois observé pour ce type de marqueur microsatellite.

Dans la pratique, l'observation d'une incohérence conduira à refaire le typage afin de vérifier la réalité de celle-ci. A titre indicatif, au cours de cette expérimentation la simple relecture de 42 gels pour lesquels une erreur de génotype avait été détectée a permis d'en corriger 24 , soit $57 \%$.

En définitive, les techniques de génétique moléculaire mises en œuvre offrent une bonne sécurité, avec un taux d'erreur de génotypage inférieur à $0,4 \%$ lorsqu'une vérification des incohérences est réalisée.

Tableau 4. Défauts d'amplification et erreurs de typage recensés par marqueur.

\begin{tabular}{|l|c|c|c|c|}
\hline Locus & Nom & $\begin{array}{c}\text { Absence } \\
\text { d'amplification }\end{array}$ & $\begin{array}{c}\text { Nombre de } \\
\text { génotypes faux }\end{array}$ & $\begin{array}{c}\text { dont allèles } \\
\text { quasi-nuls }\end{array}$ \\
\hline 1 & TGLA227 & $1 / 552(0,2 \%)$ & $3 / 547(0,5 \%)$ & $1(33 \%)$ \\
2 & INRA222 & $1 / 552(0,2 \%)$ & $4 / 547(0,7 \%)$ & $3(75 \%)$ \\
3 & INRA072 & $28 / 552(5,1 \%)$ & $16 / 520(3,1 \%)$ & $10(63 \%)$ \\
4 & ILSTS065 & $1 / 552(0,2 \%)$ & $3 / 547(0,5 \%)$ & $3(100 \%)$ \\
5 & INRA092 & $3 / 552(0,5 \%)$ & $4 / 545(0,7 \%)$ & $4(100 \%)$ \\
6 & ETH225 & $0 / 552(0 \%)$ & $1 / 548(0,2 \%)$ & $1(100 \%)$ \\
7 & INRA135 & $4 / 552(0,7 \%)$ & $2 / 544(0,4 \%)$ & $0(0 \%)$ \\
8 & INRA177 & $5 / 552(0,9 \%)$ & $0 / 543(0 \%)$ & \\
9 & BM1824 & $18 / 552(3,3 \%)$ & $6 / 53(1,1 \%)$ & $6(100 \%)$ \\
10 & HUJ1177 & $1 / 552(0,2 \%)$ & $1 / 547(0,2 \%)$ & $0(0 \%)$ \\
11 & SPS115 & $272 / 552(49,8 \%)$ & $5 / 276(1,8 \%)$ & $5(100 \%)$ \\
Total & & $\mathbf{6 2 / 5 5 2 0 ( 2 )}(\mathbf{1 , 1} \%)$ & $\mathbf{4 5 / 5 6 9 4 ( 0 , 8 \% )}$ & $\mathbf{3 3 ( 7 3 \% )}$ \\
\hline
\end{tabular}

(1) Parmi les échantillons amplifiés des 448 prélèvements utilisables.

(2) Hormis le locus 11. 


\section{3 / Qualité du dispositif de traçabilité}

La qualité du dispositif dépend en premier lieu du polymorphisme qui existe au sein de la population pour chacun des marqueurs retenus : plus celui-ci est élevé, plus est faible la probabilité de trouver deux individus ayant tous leurs allèles identiques. Un fort polymorphisme est effectivement observé pour chacun des loci étudiés, avec 5 à 12 allèles mis en évidence (tableau 5). Compte tenu des fréquences observées pour les différents allèles, il est possible de calculer la proportion d'individus dont les deux allèles sont différents à un locus donné, c'est-à-dire le degré d'hétérozygotie. Pour neuf des onze marqueurs celuici est supérieur à 0,70 , allant jusqu'à 0,87 . Sur l'ensemble des marqueurs, le taux global d'hétérozygotie est de 0,97.

Tableau 5. Nombres d'allèles identifiés et degrés d'hétérozygotie observés dans l'échantillon.

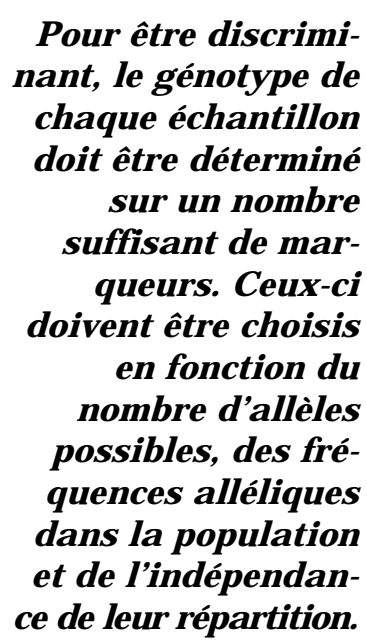

\begin{tabular}{|l|c|c|}
\hline Marqueur & Nombre d'allèles & Hétérozygotie \\
\hline TGLA 227 & 12 & 0,869 \\
INRA 222 & 11 & 0,844 \\
INRA 072 & 8 & 0,764 \\
ILSTS 065 & 8 & 0,757 \\
INRA 092 & 9 & 0,774 \\
ETH 225 & 5 & 0,703 \\
INRA 135 & 8 & 0,774 \\
INRA 177 & 11 & 0,549 \\
BM 1824 & 5 & 0,746 \\
HUJ I 177 & 7 & 0,764 \\
SPS 115 & 5 & 0,590 \\
Total & & 0,967 \\
\hline
\end{tabular}

Afin de tester l'aptitude du panel de marqueurs retenus à assurer la traçabilité des individus d'un prélèvement à l'autre, les proportions de fausses décisions ont été évaluées dans les trois situations suivantes: (a) calcul direct sur les 548 échantillons de l'expérimentation, (b) calculs théoriques sur des animaux issus d'une population dont les fréquences alléliques seraient identiques à celles observées lors de cette expérimentation, (c) calculs sur des génotypes simulés par des tirages aléatoires des allèles.

a) La traçabilité évaluée sur les 548 prélèvements a été parfaite : tous les prélèvements effectués sur un animal (poils, sang, viande) ont pu être attribués à cet animal et à lui seul. Les rares erreurs de génotypage signalées précédemment n'ont pas perturbé ces attributions.

b) A l'aide des fréquences alléliques calculées avec l'échantillon des bovins charolais du domaine de Bourges, supposé représentatif de la population charolaise, la probabilité que deux individus quelconques de cette population aient le même génotype aux 11 loci est égale à 5. $10^{-12}$ (tableau 6). La probabilité que 2 demi-frères (respectivement plein frères) de cette population aient le même génotype aux 11 loci est de 5,7. 10 ${ }^{-9}$ (respecti- vement 4,6. $\left.10^{-5}\right)$. Ces quantités extrêmement faibles dénotent une grande puissance du dispositif étudié.

Tableau 6. Probabilités que deux individus aient le même génotype.

\begin{tabular}{|l|c|c|c|}
\hline Individus & 11 locus & 8 locus & 5 locus \\
\hline non apparentés & $5,0.10^{-12}$ & $7,5 \cdot 10^{-10}$ & $7,2 \cdot 10^{-5}$ \\
demi-frères & $5,7.10^{-9}$ & $1,9.10^{-7}$ & $9,8 \cdot 10^{-4}$ \\
plein frères & $4,6.10^{-5}$ & $3,9.10^{-4}$ & $1,9.10^{-2}$ \\
\hline
\end{tabular}

Si le nombre de loci est plus faible, la probabilité d'avoir deux génotypages identiques pour deux individus différents augmente. A l'évidence, 5 loci sont insuffisants pour identifier convenablement un bovin charolais car il existe un risque d'environ 1/14 000 que deux veaux charolais aient des allèles identiques à ces cing loci.

c) Dix mille couples de génotypes à 11 loci ont été simulés, sous chacune des deux hypothèses qui ont fait l'objet de cette étude : $\mathrm{H} 0=$ même individu ou $\mathrm{H} 1=$ deux individus différents. Quelques erreurs de génotype ont été introduites aléatoirement dans une proportion de $1 \%$ d'allèles faussement déterminés, ce qui est bien supérieur à ce qui a été observé dans le cadre de notre expérience.

Le test de maximum de vraisemblance montre que tous les individus différents ont été déclarés comme tels par le test statistique, c'est-à-dire qu'il n'y a pas un seul cas parmi les 10000 où l'hypothèse HO soit acceptée alors que I'hypothèse $\mathrm{H} 1$ était vraie.

Lorsque la simulation a été réal isée sous I'hypothèse $H 0$, il n'y a eu que 3 cas sur les 10000 soit $0,03 \%$, où les deux échantillons ont été déclarés provenir de deux individus différents alors qu'ils provenaient d'un seul et même individu. Cette occurrence, bien que calculée avec un taux d'erreur de génotypage de $1 \%$, est très faible. Comme dans la pratique les génotypes litigieux seront systématiquement déterminés à nouveau, il peut être retenu que l'immense majorité des ambiguïtés seront levées. Cette fréquence de $0,03 \%$ est donc à considérer comme la fréquence maximale de l'erreur qui est faite lorsqu'on déclare que deux échantillons proviennent de deux individus différents alors qu'il ont été prélevés sur le même animal.

En conclusion de cette partie, des suggestions peuvent être faites concernant le nombre minimum de marqueurs nécessaire pour assurer une traçabilité quasi parfaite. Avec les fréquences d'allèles obtenues dans la présente expérimentation, il apparaît que huit loci (convenablement choisis sur la base d'un fort polymorphisme) sont nécessaires et qu'en decà, le nombre d'erreurs de décision deviendrait inacceptable. Ce nombre de loci est plus élevé que celui avancé par Meghen et al (1998). Le choix des marqueurs doit reposer sur le degré d'hétérozygotie moyenne 
comme mesure du polymorphisme plutôt que sur le nombre d'allèles, car l'existence d'allèles nombreux mais rares n'apporte guère un surcroît de puissance.

\section{Conclusion}

Tous les types de prélèvements étudiés (poils, sang, viande au scalpel ou à l'aiguille à biopsie 24 heures ou 15 jours après abattage) ont donne de bons résultats en terme de qualité de l'ADN extrait, à quelques nuances près. En particulier, le scalpel pourra être remplacé par l'aiguille à biopsie sans grand risque de perte d'information. Suite à cette étude, des analyses préliminaires ont été pratiquées sur la viande après cuisson (micro-ondes, poêle). L'obtention, même dans ces conditions, d'ADN de bonne qualite renforce encore l'intérêt de notre approche.

Les rares erreurs de génotypage liées aux marqueurs microsatellites sont une réalité et il serait malhonnête de passer cet aspect sous silence. Pour la première fois sont publiés ici les divers aspects de la qualité des typages effectués à LABOGENA, en terme de qualité de I'ADN et de l'amplification PCR, selon le type d'échantillon. Les rares erreurs comptabilisées n'ont pas mis en cause la démarche générale puisque le modèle statistique les prend en considération, et que, de toute façon, elles sont levées à la moindre incohérence observée par un nouveau typage.

Des erreurs d'étiquetage d'échantillons lors de l'expérimentation ont été mises en évidence. Ceci souligne encore les bonnes performances de ce dispositif de traçabilité (méthodologies moléculaire et statistique), mais permet aussi d'attirer l'attention sur la gestion des échantillons qui devra être extrêmement rigoureuse et sans faille, si ce type de suivi des viandes bovines est mis en place à grande échelle. De plus cela apporte une preuve supplémentaire de l'intérêt de réaliser de tels types de contrôle au cours de la chaîne pour en vérifier la fiabilité.

II faut souligner que les divers prélèvements furent effectués dans des domaines ou abattoir expérimentaux de I'INRA et que, pour une utilisation en élevage, en abattoir ou en atelier de découpe, il est primordial d'accorder la plus grande importance à la qualité des prélèvements afin d'éviter toute contamination par du matériel génétique provenant d'autres animaux, ce qui pourrait fausser les résultats.
Pour conclure, la faisabilité d'une méthode de traçabilité individuelle des viandes grâce à des marqueurs moléculaires de type microsatellite a été prouvée en toute rigueur dans cette étude. En particulier cette méthode est particulièrement apte à détecter toute incompatibilité d'identification entre une pièce de viande et l'animal dont elle est supposée provenir. La puissance du dispositif a eté évaluée selon le nombre de marqueurs utilisés en montrant l'intérêt de marqueurs polymorphes avec un degré élevé d'hétérozygotie.

La mise en place d'une traçabilité quasi parfaite des animaux et des viandes dans la filière viande bovine ne dépend donc plus de la maîtrise des techniques de génétique moléculaire, mais de l'organisation de cette filière pour prélever les animaux, extraire l'ADN, le stocker et le génotyper. II est possible d'imaginer différents scénarios plus ou moins contraignants. Une solution maximaliste serait de constituer une banque d'ADN systématique pour tout bovin né ou élevé en France parallèlement à celle générée par I'IPG. Pour ceci il serait nécessaire de prévoir le prélèvement systématique d'un tissu (poil, morceau d'oreille) lors de l'identification de l'animal (pose des boucles). Une solution moins systématique pourrait consister à ne réaliser ces extractions d'ADN et ces génotypages que lorsqu'il y a nécessité de vérifier l'information portée sur une étiquette. Pour ceci il est nécessaire de disposer d'un prélèvement de tissu dont l'origine ne puisse être mise en doute. Le prélèvement puis le stockage par l'abattoir de l'ensemble oreille / boucle d'identification de tout animal abattu pourrait servir de preuve irréfutable de la liaison animal / viande lorsque le génotypage sera effectué.

L'essentiel des problèmes à résoudre pour mettre en place une traçabilité individuelle des viande a l'aide de marqueurs moléculaires concerne des aspects de logistique, d'organisation et de volonté des opérateurs de la filière.

\section{Remerciements}

Nous remercions toutes les personnes qui ont contribué à l'obtention des échantillons et à l'acquisition des résultats présentés, en particulier les collègues des domaines INRA de Bourges et du Pin-au-Haras, ainsi que la société INTERBEV et le CIV pour leur soutien financier (contrat B 01536).

\section{Références}

Balding D.J ., Nichols R.A., 1995. A method for quantifying differentiation between populations at multi-allelic loci and its implications for investigating identity and paternity. Genetica, 96, 3-12.

Chaisemartin D., 1998. Bovins, ovins, caprins, porcins. La traçabilité est aussi une contrainte réglementaire. Viandes Prod. Carnés, 19, 9-14.

Combris P., 1997. La consommation des produits animaux en France : tendances et perspectives d'évolution. INRA Prod. Anim., 10, 267-274.
Cornuet J .M., Aulagnier S., Lek S., Franck P., Solignac M., 1996. Classifying individuals among infra-specific taxa using microsatellite data and neural networks. C.R. Acad. Paris, Sciences de la vie/Life sciences, 319,1167-1177.

Dupit J., 1998. Abattage et découpe des viandes bovines. Les nouvelles contraintes exigent des réorganisations du travail. Viandes Prod. Carnés, 19, 25-30.

Léonard M., Kirszenbaum M., Cotinot $C$., Chesné $P$. ., Heyman Y., Stinnakre M.G., Bishop C., Delouis C., Vaiman 
M., Fellous M., 1987. Sexing bovine embryos using $Y$ chromosome specific DNA probe. Theriogenology, 27, 248.

Meghen C.N, Scott C.S., Bradley D.G., MacHugh D.E., Loftus R.T., Cunningham E.P., 1998. DNA based traceability techniques for the meat industry. Anim. Genet., 29 (Suppl. 1), 48-49.

Moazami-Goudarzi K., Laloë D., Furet J .P., Grosclaude F., 1997. Analysis of genetic relationsips between 10 cattle breeds with 17 microsatellites. Anim. Genetics, 28, 338-345.

Ollivier L., Chevalet C., Foulley J.L., 2000. Utilisation des marqueurs pour la caractérisation des ressources génétiques. INRA Prod. Anim., numéro hors série «Génétique moléculaire : principes et application aux populations animales», 247-252.

Potherat C., Mainsant P., 1995. Le recul des viandes de ruminants dans la consommation de viandes, volailles et poisson en France au cours des 25 dernières années : une réorientation du choix des ménages. Renc. Rech. Ruminants, 2, 1-8.

Renou J.P., 1998. Les méthodes analytiques. La dilution isotopique caractérise l'origine d'un produit. Viandes Prod. Carnés, 19, 77.

Rouzaud F., Martin J ., Gallet F., Delourme D., Petit J M. Levéziel H., J ulien R., Oulmouden A., 2000. Utilisation de marqueurs génétiques pour la traçabilité. Intérêt des gènes de la coloration de la robe chez le bovin. INRA Prod. Anim., numéro hors série «Génétique moléculaire : principes et application aux populations animales», 243245

Thonat C., Viallon C., Berdagué J.L., 1999 Caractérisation et différentiation de produits par analyse des composés volatils. Ann. Fals. Exp. Chim. 92, n948, 365-371.

\begin{abstract}
Individual traceability of bovine meat using molecular markers.

In response to the confidence crisis of consumers and their regular disinterest on bovine meat, within the framework of efforts that are now developed to improve and secure bovine traceability, our aim was the proposal and validation of a method of individual identification of bovine meat via microsatellite molecular markers. Several samples were taken in vivo and post mortem from young cattle of INRA experimental farms. The 11 loci genotype determination, then the two-bytwo comparison of these genotypes using an adequate
\end{abstract}

Abstract

statistical method, taking into account the microsatellite particularities, allowed us to propose some advises on the typing effort (marker number) necessary for assuring a perfect traceability on one hand, and on essential precautions to be taken in large scale implementation, i.e. sample management, on the other hand.

SANCRISTOBAL-GAUDY M., RENAND G., AMIGUES Y., BOSCHER M.-Y., LEVEZIE L H., BIBE B., 2000. Traçabilité individuelle des viandes bovines à l'aide de marqueurs génétiques. INRA Prod. Anim., 13, 269-276. 